\title{
Study of the asymptotic eigenvalue distribution and trace formula of a second order operator- differential equation
}

Nigar Mahar Aslanova ${ }^{1,2}$

Correspondence: nigar. aslanova@yahoo.com

'Department of Differential Equation, Institute of Mathematics and Mechanics-Azerbaijan National Academy of Science, 9, F. Agayev Street, Baku AZ1141, Azerbaijan Full list of author information is available at the end of the article

\begin{abstract}
The purpose of writing this article is to show some spectral properties of the Bessel operator equation, with spectral parameter-dependent boundary condition. This problem arises upon separation of variables in heat or wave equations, when one of the boundary conditions contains partial derivative with respect to time. To illustrate the problem and the proof in detail, as a first step, the corresponding operator's discreteness of the spectrum is proved. Then, the nature of the eigenvalue distribution is established. Finally, based on these results, a regularized trace formula for the eigenvalues is obtained.
\end{abstract}

MSC: 34B05; 34G20; 34L20; 34L05; 47A05; 47A10.

Keywords: Hilbert space, discrete spectrum, regularized trace

\section{Introduction}

Let $\mathbf{L}_{2}=L_{2}(H,[0,1]) \oplus H$, where $H$ is a separable Hilbert space with a scalar product $(\cdot, \cdot)$ and a norm $\|\cdot\|$ inside of it. By definition, a scalar product in $\mathbf{L}_{2}$ is

$$
(Y, Z)_{\mathbf{L}_{2}}=\int_{0}^{1}(\gamma(t), z(t)) d t-\frac{1}{h}\left(y_{1}, z_{1}\right), \quad h<0,
$$

where $Y=\left\{y(t), y_{1}\right\}, Z=\left\{z(t), z_{1}\right\}$ and $y(t), z(t) \in L_{2}(H,[0,1])$ for which $L_{2}(H,[0$, $1])$ is a space of vector functions $y(t)$ such that $\int_{0}^{1}\|y(t)\|^{2} d t<\infty$.

Now, consider the equation:

$$
\begin{aligned}
& l[y] \equiv-\gamma^{\prime \prime}(t)+\frac{v^{2}-\frac{1}{4}}{t^{2}} y(t)+A y(t)+q(t) y(t)=\lambda y(t), \quad t \in(0,1), v \geq 1, \\
& y(1)-h y^{\prime}(1)=\lambda y(1)
\end{aligned}
$$

in $L_{2}(H,[0,1])$, where $A$ is a self-adjoint positive-definite operator in $H$ which has a compact inverse operator. Further, suppose the operator-valued function $q(t)$ is weakly measurable, and $\|q(t)\|$ is bounded on $[0,1]$ with the following properties:

1. $q(t)$ has a second-order weak derivative on $[0,1]$, and $q^{(l)}(t)(l=0,1,2)$ are selfadjoint operators in $H$ for each $t \in[0,1],\left[q^{(l)}(t)\right]^{*}=q^{(l)}(t), q^{(l)}(t) \in \sigma_{1}(H)$. Here

\section{SpringerOpen ${ }^{\circ}$}

(C) 2011 Aslanova; licensee Springer. This is an Open Access article distributed under the terms of the Creative Commons Attribution License (http://creativecommons.org/licenses/by/2.0), which permits unrestricted use, distribution, and reproduction in any medium, provided the original work is properly cited. 
$\sigma_{1}(H)$ is a trace class, i.e., a class of compact operators in separable Hilbert space $H$, whose singular values form a convergent series (denoting the compact operator by $B$, then its singular values are the eigenvalues of $\left(B B^{*}\right)^{\left.\frac{1}{2}\right)}$. If $\left\{\phi_{n}\right\}$ is a basis formed by the orthonormal eigenvectors of $B$, then $\|B\|_{\sigma_{1}(H)}=\sum\left|\left(B \varphi_{n}, \varphi_{n}\right)\right|$. For simplicity, denote the norm in $\sigma_{1}(H)$ by $\|\cdot\|_{1}$.

2. The functions $\left\|q^{(l)}(t)\right\|_{1}(l=0,1,2)$ are bounded on $[0,1]$.

3. The relation $\int_{0}^{1}(q(t) f, f) d t=0$ is true for each $f \in H$.

State that if $q(t) \equiv 0$, a self-adjoint operator denoted by $L_{0}$ can be associated with problem (2), (3) whose definition will be given later.

If $q(t) \otimes 0$, the operators $L$ and $Q$ are defined by $L=L_{0}+Q$, and $Q: Q\left\{y(t), y_{1}\right\}=\{q$ ( $t) y(t), 0\}$ which is a bounded self-adjoint operator in $\mathbf{L}_{2}$.

After the above definitions and the assumptions, the asymptotic of the eigenvalue distribution and regularized trace of the considered problem will be studied. It is clear that because of the appearance of an eigenvalue parameter in the boundary condition at the end point, the operator associated with problem (2), (3) in $L_{2}(H,[0,1])$ is not self-adjoint. Introduce a new Hilbert space $L_{2}(H,[0,1]) \oplus H$ with the scalar product defined by formula (1) similar to one used in [1]. Then, in this space, the operator becomes self-adjoint.

In [2], Walter considers a scalar Sturm-Liouville problem with an eigenvalue parameter $\lambda$ in the boundary conditions. He shows that one can associate a self-adjoint operator with that by finding a suitable Hilbert space. Further, he obtains the expansion theorem by reference to the self-adjointness of that operator. His approach was used by Fulton in [3] later on.

As for the differential operator equations, to the best of this author's knowledge in the articles [1,4-6], an eigenvalue parameter appears in the boundary conditions. In [4], the following problem is considered:

$$
\begin{gathered}
-u^{\prime \prime}(x)+A u(x)=\lambda u(x), \quad x \in(0, b), \\
u^{\prime}(0)+\lambda u(0)=0, \quad u(b)=0,
\end{gathered}
$$

where $A=A^{*}>E$, and $u(x) \in L_{2}(H,(0, b))$. It is proved that the operator associated with this problem has a discrete spectrum, iff : $A$ has a discrete spectrum. The eigenvalues of this problem form two sequences like $\lambda_{k} \sim \sqrt{\mu_{k}}$ and $\lambda_{m, k}=\mu_{k}+\frac{n^{2} \pi^{2}}{b^{2}}$ where $n, k$ $\in N$, and $\mu_{k}$ is an eigenvalue of $A$. This is obtained from appearance of $\lambda$ in the boundary condition.

In [5], both boundary conditions depend on $\lambda$. It is shown that the operator defined in the space $L_{2}(H,(0,1)) \oplus H \oplus H$ is symmetric positive-definite. Further, the asymptotic formulas for eigenvalues are obtained.

In this author's previous study [6], for the operator considered in [4], the trace formula has been established.

If $h=0$ in (3), then the boundary condition takes the form $y(1)=0$. This problem is considered in [[7], Theorem 2.2], where the trace formula is established. It is proved that there exists a subsequence of natural numbers $\left\{n_{m}\right\}$ such that 
$\lim _{m \rightarrow \infty} \sum_{n=1}^{n_{m}}\left(\mu_{n}-\lambda_{n}\right)=-\frac{2 v \operatorname{trq}(0)+\operatorname{trq}(1)}{4}$, where $\mu_{n}$ and $\lambda_{n}$ are the eigenvalues of perturbed and non-perturbed operators. For definition of $\left\{n_{m}\right\}$, see also [[8], Lemma 1].

For a scalar case, please refer to [9], where the following problem

$$
\begin{gathered}
-y^{\prime \prime}+\frac{v^{2}-\frac{1}{4}}{x^{2}} y+q(x) y=\lambda^{2} y, \\
y(\pi)=0
\end{gathered}
$$

is considered on the interval $[0, \pi]$. Then, the sum $\sum_{n=1}^{\infty}\left(\lambda_{n}-\left(n+\frac{v}{2}-\frac{1}{2}\right)^{2}\right)$ is calculated.

In comparison with the above mentioned articles, here we consider a differential operator equation which has a singularity at 0 , and the boundary condition at 1 involves both the eigenvalue parameter $\lambda$ and physical parameter $h<0$.

Problems with $\lambda$-dependent boundary conditions arise upon separation of variables in the heat and wave equations. We can also refer to [10-17], where boundary-value problems for ordinary differential operators with eigenvalue-dependent boundary conditions are studied.

In 1953, Gelfand and Levitan [18] considered the Sturm-Liouville operator

$$
-y^{\prime \prime}(x)+q(x) y(x)=\lambda y(x), \quad y^{\prime}(0)=0, \quad y^{\prime}(\pi)=0, \quad q(x) \in C^{\prime}[0, \pi]
$$

and derived the formula $\sum_{n=1}^{\infty}\left(\mu_{n}-\lambda_{n}\right)=\frac{1}{4}(q(0)+q(\pi))$, where $\mu_{n}$ are the eigenvalues of the above operator. For $q(x) \equiv 0$ the eigenvalues of the operator are given by $\lambda_{n}=n^{2}$.

It is worthwhile to note that, several studies are devoted to searching a regularized trace for the concrete operators (e.g., [9-18]), as well as differential-operator equations (e.g., [6-8,19]) and discrete abstract operators (e.g., [20-22]). For further detailed discussion of the subject, please refer to [23].

Trace formulas are used for the approximation of the first eigenvalues of the operators $[24,25]$ to solve inverse problems $[26,27]$. They are also applied to index theory of linear operators $[28,29]$.

To summarize this study, in Section 1, it is proved that the operator associated with (2), (3) is self-adjoint and has a discrete spectrum. In Section 2, we establish an asymptotic formula for the eigenvalues. To do this, the zeros of the characteristic equation (Lemmas 2.1, 2.2, 2.3) are searched in detail. In Section 3, by using the asymptotic for the eigenvalues, we prove that the series called "a regularized trace" converges absolutely (Lemma 3.1). This enables us to arrange the terms of the series in a suitable way for calculation as in (3.9). To calculate the sum of this series, we introduce a function whose poles are zeros of the characteristic equation, the residues at poles of which are the terms of our series. Finally, we establish a trace formula by integrating this function along the expanded contours.

In conclusion, we apply the results of our study to a boundary value problem generated by a partial differential equation. 


\section{Definition of $L_{0}$ and proof of discreteness of the spectrum}

Let $D\left(L_{0}^{\prime}\right)=\left\{Y: Y=\{y(t), y(1)\}, y(t) \in C_{0}^{\infty}(H,[0,1]), \gamma(t) \in D(A)\right\}$, where $C_{0}^{\infty}(H,[0,1])$ is a set of vector functions with values in $H$ (see [30], p. 57) that vanish in the vicinity of zero and are infinitely differentiable in the norm of $H$. Also, on $D\left(L_{0}^{\prime}\right)$ define the operator $L_{0}^{\prime}$ :

$$
L_{0}^{\prime} Y=\left\{l[y], y(1)-h \gamma^{\prime}(1)\right\} .
$$

Using integration by parts it is easy to see that $L_{0}^{\prime}$ is symmetric. Denote its closure by $L_{0}$ and show that it is self-adjoint. To do that, consider the adjoint operator of $L_{0}^{\prime}$ as $L_{0}^{\prime *}$. By definition, vector $Z=\left\{z(t), z_{1}\right\} \in D\left(L_{0}^{\prime *}\right)$ if for each $Y \in D\left(L_{0}^{\prime}\right)$ it holds

$$
\int_{1}^{1}(l([y], z(t))) d t-\frac{1}{h}\left(y(1)-h \gamma^{\prime}(1), z_{1}\right)=\int_{0}^{1}\left(y(t), z^{*}(t)\right) d t+\left(y(1), z^{*}\right)
$$

and $Z^{*}=\left\{z^{*}(t), z^{*}\right\} \in \mathbf{L}_{2}$. However, using integration by parts from (1.1), it is obvious that $D\left(L_{0}^{\prime *}\right)=\left\{Z: Z=\left\{z(t), z_{1}\right\} \in \mathbf{L}_{2}\right.$ with $z(t) \in W_{2}^{2}(H,[0,1])$ and $l[z] \in L_{2}$ $(H,[0,1])\}$. In other words, $z(t)$ has a first-order derivative on $[0,1]$ which is absolutely continuous in the norm of $H$ and $z(0)=z^{\prime}(0)=0, A z(t) \in L_{2}(H,[0,1])$ and $Z^{*}=L_{0}^{\prime *} Z=\left\{l[z], z(1)-h z^{\prime}(1)\right\}$.

Now, the vector $Z \in D\left(L_{0}^{\prime * *}\right)$ if and only if for any $Y \in D\left(L_{0}^{\prime *}\right)(1.1)$ holds, $Z^{*} \in \mathbf{L}_{2}$ and $Z^{*}=L_{0}^{\prime * *} Z$.

By virtue of $L_{0}^{\prime} \subset L_{0}^{\prime *}, L_{0}^{\prime * *} \subseteq L_{0}^{\prime *}$, we can state that any vector $Z$ from $D\left(L_{0}^{\prime * *}\right)$ must also belong to $D\left(L_{0}^{\prime *}\right)$ and $L_{0}^{\prime * *} Z=L_{0}^{\prime *} Z$. On the other hand, it could be verified that relation (1.1) is also true for

$$
\begin{gathered}
Y \in D\left(L_{0}^{\prime}{ }^{*}\right), \quad Z(t) \in W_{2}^{2}(H,[0,1]), \\
l[z] \in L_{2}(H,[0,1]) Z^{*}=\left\{l[z], z(1)-h z^{\prime}(1)\right\} .
\end{gathered}
$$

Therefore, $L_{0}^{\prime * *}=L_{0}^{\prime *}$. In other words, $L_{0}^{\prime *}$ is a self-adjoint operator. However, we know that $L_{0}^{\prime * *}=\bar{L}_{0}^{\prime}$. Thus, the closure of $L_{0}^{\prime}$ is a self-adjoint operator $L_{0}^{\prime *}$, which we will denote by $L_{0}$.

By virtue of all as stated above, $L_{0}$ is defined as

$$
\begin{gathered}
D\left(L_{0}\right)=\left\{Y \in \mathbf{L}_{2}, \gamma^{\prime \prime}(t), A y(t) \in L(H,(0,1)), \gamma_{1}=\gamma(1)\right\}, \\
L_{0} \gamma=\left\{l[y], y(1)-h \gamma^{\prime}(1)\right\} .
\end{gathered}
$$

By the properties of $v \geq 1, A>E$, it follows that $L_{0}$ is a positive-definite operator. To show that, for each $Y \in D\left(L_{0}\right)$, we have

$$
\begin{aligned}
\left(L_{0} Y, Y\right)_{\mathbf{L}_{2}} & =\int_{0}^{1}(l[y], y(t)) d t-\frac{1}{h}\left(y(1)-h y^{\prime}(1), \gamma(1)\right) \\
& =\int_{0}^{1}\left\|y^{\prime}(t)\right\|^{2} d t+\int_{0}^{1}(A y(t), y(t)) d t+\int_{0}^{1} \frac{v^{2}-\frac{1}{4}}{t^{2}}\|y(t)\|^{2} d t-\frac{1}{h}\|y(1)\|^{2} \\
& \geq \int_{0}^{1}\left\|y^{\prime}(t)\right\|^{2} d t+\int_{0}^{1}\|y(t)\|^{2} d t .
\end{aligned}
$$

Since the embedding $W_{2}^{1}(H,(0,1)) \subset C(H,[0,1])$ is continuous ([[31], Theorem 1.7.7], [[32], p. 48]), then, $\|y(1)\| \leq c\|y(t)\|_{W_{2}^{1}(H,(0,1))}$, where $c>0$ is a constant. 
Thus,

$$
\left(L_{0} Y, Y\right)_{\mathbf{L}_{2}} \geq C\left(\int_{0}^{1}\|y(t)\|^{2} d t-\frac{1}{h}\|y(1)\|^{2}\right)=C\|Y\|_{\mathbf{L}_{2}}^{2}
$$

which shows that $L_{0}$ is a positive-definite operator.

To prove the discreteness of the spectrum, we will use the following Rellich's theorem (see [[33], p. 386]).

Theorem 1.1. Let $B$ be a self-adjoint operator in $H$ satisfying $(B \phi, \phi) \geq(\phi, \phi), \phi \in$ $D_{B}$, where $D_{B}$ is a domain of $B$.

Then, the spectrum of $B$ is discrete if and only if the set of all vectors $\phi \in D_{B}$, satisfying $(B \phi, \phi) \leq 1$ is precompact.

Let $\gamma_{1} \leq \gamma_{2} \leq \cdots \leq \gamma_{n} \leq \cdots$ be the eigenvalues of $A$ counted with multiplicity and $\phi_{1}$, $\phi_{2}, \ldots, \phi_{n}, \ldots$ be the corresponding orthonormal eigenvectors in $H$.

Take $y_{k}(t)=\left(y(t), \phi_{k}\right)$. Then

$$
\begin{gathered}
(y(t), \gamma(t))=\sum_{k=1}^{\infty}\left|\gamma_{k}(t)\right|^{2}, \\
\left(\left(\frac{v^{2}-\frac{1}{4}}{t^{2}} E+A\right) \gamma, y\right)=\sum_{k=1}^{\infty}\left(\frac{v^{2}-\frac{1}{4}}{t^{2}}+\gamma_{k}\right)\left|\gamma_{k}(t)\right|^{2} .
\end{gathered}
$$

Hence, using the Rellich's theorem, we come to the following theorem:

Theorem 1.2. If the operator $A^{-1}$ is compact in $H$, then the operator $L_{0}$ has a discrete spectrum.

Proof. By virtue of positive-definiteness of $L_{0}$, by Rellich's theorem, it is sufficient to show that the set of vectors

$$
\begin{gathered}
\mathbf{Y}=\left\{Y \in D\left(L_{0}\right) \backslash\left(L_{0} Y, Y\right)_{\mathbf{L}_{2}}\right. \\
=\int_{0}^{1}\left[\left(\gamma^{\prime}(t), \gamma^{\prime}(t)\right)+\left(\left(\frac{v^{2}-\frac{1}{4}}{t^{2}} E+A\right) \gamma(t), \gamma(t)\right)\right] d t \\
\left.-\frac{1}{h}(\gamma(1), \gamma(1)) \leq 1\right\}
\end{gathered}
$$

is precompact in $L_{2}$.

To prove this theorem, consider the following lemma.

Lemma 1.1. For any given $\varepsilon>0$, there is a number $R=R(\varepsilon)$, such that

$$
\int_{0}^{1} \sum_{k=R+1}^{\infty}\left|y_{k}(t)\right|^{2} d t-\frac{1}{h} \sum_{k=R+1}^{\infty}\left|y_{k}(1)\right|^{2}<\varepsilon .
$$

Proof. From (1.1) for $Y \in \mathbf{Y}$ :

$$
\begin{gathered}
\int_{0}^{1} \sum_{k=R+1}^{\infty}\left|\gamma_{k}(t)\right|^{2} d t=\frac{1}{\gamma_{R}} \int_{0}^{1} \sum_{k=R+1}^{\infty}\left|\gamma_{k}(t)\right|^{2} \gamma_{R} d t \leq \frac{1}{\gamma_{R}} \int_{0}^{1} \sum_{k=R+1}^{\infty}\left|y_{k}(t)\right|^{2} \gamma_{k} d t \\
\leq \frac{1}{\gamma_{R}} \int_{0}^{1} \sum_{k=1}^{\infty}\left|\gamma_{k}(t)\right|^{2} \gamma_{k} d t=\frac{1}{\gamma_{R}} \int_{0}^{1}(A y, y) d t \leq \frac{1}{\gamma_{R}}\left(L_{0} Y, Y\right)_{\mathrm{L}_{2}} \leq \frac{1}{\gamma_{R}} .
\end{gathered}
$$


Since $\gamma_{R} \rightarrow \infty$ for $R \rightarrow \infty$, for any given $\varepsilon>0$, we could choose $R(\varepsilon)$ such that $\frac{1}{\gamma_{R}}<\frac{\varepsilon^{2}}{\left(1-\frac{2}{h}\right)^{2}}$. Therefore, for this choice of $R$ the inequality

$$
\int_{0}^{1} \sum_{k=R+1}^{\infty}\left|y_{k}(t)\right|^{2} d t<\frac{\varepsilon^{2}}{\left(1-\frac{2}{h}\right)^{2}}
$$

holds. On the other hand, by virtue of (1.3):

$$
\begin{aligned}
- & \frac{1}{h} \sum_{k=R+1}^{\infty}\left|y_{k}(1)\right|^{2}=-\frac{1}{h} \sum_{k=R+1}^{\infty}\left|\int_{0}^{1}\left(y_{k}^{2}(t)\right)^{\prime} d t\right|=-\frac{1}{h} \sum_{k=R+1}^{\infty}\left|\int_{0}^{1} 2 \gamma_{k}^{\prime}(t) y_{k}(t) d t\right| \\
& \leq-\frac{2}{h} \sum_{k=R+1}^{\infty}\left(\int_{0}^{1}\left|y_{k}^{\prime}(t)\right|^{2} d t\right)^{1 / 2}\left(\int_{0}^{1}\left|y_{k}(t)\right|^{2} d t\right)^{1 / 2} \\
& \leq-\frac{2}{h}\left(\sum_{k=R+1}^{\infty} \int_{0}^{1}\left|y_{k}^{\prime}(t)\right|^{2} d t\right)^{1 / 2}\left(\sum_{k=R+1}^{\infty} \int_{0}^{1}\left|y_{k}(t)\right|^{2} d t\right)^{1 / 2} \leq \frac{-2}{\sqrt{\gamma_{R}} h}<-\frac{2}{h} \cdot \frac{\varepsilon}{1-\frac{2}{h}} .
\end{aligned}
$$

From (1.4) and the above, it follows that

$$
\int_{0}^{1} \sum_{k=R+1}^{\infty}\left|y_{k}(t)\right|^{2} d t-\frac{1}{h} \sum_{k=R+1}^{\infty}\left|y_{k}(1)\right|^{2}<\frac{\varepsilon^{2}}{\left(1-\frac{2}{h}\right)^{2}}-\frac{\frac{2}{h} \varepsilon}{1-\frac{2}{h}}<\frac{\varepsilon}{\left(1-\frac{2}{h}\right)}-\frac{\frac{2}{h} \varepsilon}{1-\frac{2}{h}}=\varepsilon .
$$

This proves Lemma 1.1.

Now, turn to the proof of Theorem 1.2. Assume, $Y \in \mathbf{Y}$. Denote the set of all vector-functions $\tilde{Y}=\left\{\sum_{k=1}^{R} \gamma_{k}(t) \varphi_{k}, \sum_{k=1}^{R} \gamma_{k}(1) \varphi_{k}\right\} \in \mathbf{L}_{2}$, by $E_{R}$. Then, from Lemma 1.1 it follows that for the set $Y, E_{R}$ is an $\varepsilon$-net in $\mathbf{L}_{2}$. Therefore, to prove the precompactness of the set $\mathbf{Y}$, we must prove the precompactness of $E_{R}$ in $\mathbf{L}_{2}$. Since $\left|y_{k}(1)\right| \leq 1$ $(k=1, \ldots, R)$, it is sufficient to show that $y_{k}(t)(k=1, \ldots, R)$ satisfies the criteria of precompactness in $L_{2}(0,1)$ [[34], p. 291]. In other words, $y_{k}(t),(k=1, \ldots, R)$ must be equicontinuous and bounded with respect to the norm in $L_{2}(0,1)$. To show that, using (1.3) results in

$$
\int_{0}^{1}\left|y_{k}(t)\right|^{2} d t \leq \int_{0}^{1}(y(t), y(t)) d t \leq \int_{0}^{1}(A y, y) d t \leq 1
$$

which proves the boundedness of the functions $y_{k}(t)(k=1, \ldots, R)$. Assume that $y_{k}(t)$ is a zero outside the interval $(0,1)$. Then, by using the following relation

$$
\left|\gamma_{k}(t+\eta)-y_{k}(t)\right| \leq \int_{0}^{\eta}\left|y_{k}^{\prime}(t+\xi)\right| d \xi
$$

we have

$$
\int_{0}^{1}\left|y_{k}(t+\eta)-y_{k}(t)\right|^{2} d t \leq \int_{0}^{1-\eta}\left|y_{k}(t+\eta)-y_{k}(t)\right|^{2} d t+\int_{1-\eta}^{1}\left|y_{k}(t)\right|^{2} d t
$$




$$
\begin{aligned}
& \int_{1-\eta}^{1}\left|\gamma_{k}(t)\right|^{2} d t=\int_{1-\eta}^{1}\left|\int_{0}^{t} \gamma_{k}^{\prime}(\tau) d \tau\right|^{2} d t \leq \int_{1-\eta}^{1}\left|\int_{0}^{1} \gamma_{k}{ }_{k}(\tau) d \tau\right|^{2} d t \leq \eta \\
& \int_{0}^{1-\eta}\left|y_{k}(t+\eta)-\gamma_{k}(t)\right|^{2} d t \leq \int_{0}^{1-\eta}\left(\int_{0}^{\eta}\left|\gamma_{k}^{\prime}(\tau+t)\right| d \tau\right)^{2} d t \\
& \leq \int_{0}^{1-\eta} \eta \int_{0}^{\eta}\left|y^{\prime}{ }_{k}(\tau+t)\right|^{2} d \tau d t \leq \int_{0}^{1-\eta} \eta \int_{0}^{1}\left|\gamma^{\prime}(\tau)\right|^{2} d \tau d t<\eta(1-\eta)<\eta .
\end{aligned}
$$

From the above, for $|\eta|<\varepsilon$ we have

$$
\int_{0}^{1}\left|y_{k}(t+\eta)-y_{k}(t)\right|^{2} d t<2 \varepsilon .
$$

This shows the equicontinuity of $E_{R}$, and it completes the proof of the discreteness of the spectrum of $L_{0}$.

\section{The derivation of the asymptotic formula for eigenvalue distribution of $L_{\mathbf{0}}$}

Suppose that the eigenvalues of $A$ are $\gamma_{n} \sim a n^{\alpha}(n \rightarrow \infty, a>0, \alpha>0)$. Then, by virtue of the spectral expansion of the self-adjoint operator $A$, we get the following boundary value problem for the coefficients $y_{k}(t)=\left(y(t), \phi_{k}\right)$ :

$$
\begin{aligned}
& -\gamma_{k}^{\prime \prime}(t)+\frac{v^{2}-\frac{1}{4}}{t^{2}} y_{k}(t)=\left(\lambda-\gamma_{k}\right) \gamma_{k}(t), \quad t \in(0,1), \\
& y_{k}(1)-h \gamma_{k}^{\prime}(1)=\lambda \gamma_{k}(1) .
\end{aligned}
$$

The solution to problem $(2.1)$ from $L_{2}(0,1)$ is

$$
\gamma_{k}(t)=\sqrt{t} J_{v}\left(t \sqrt{\lambda-\gamma_{k}}\right) .
$$

For this solution to satisfy (2.2), it is necessary and sufficient to hold

$$
J_{v}\left(\sqrt{\lambda-\gamma_{k}}\right)-\frac{h}{2} J_{v}\left(\sqrt{\lambda-\gamma_{k}}\right)-h \sqrt{\lambda-\gamma_{k}} J_{v}^{\prime}\left(\sqrt{\lambda-\gamma_{k}}\right)-\lambda J_{v}\left(\sqrt{\lambda-\gamma_{k}}\right)=\text { (2.3) }
$$

at least for one $\gamma_{k}\left(\lambda \neq \gamma_{k}\right)$. Therefore, the spectrum of the operator $L_{0}$ consists of those real values of $\lambda \neq \gamma_{k}$, such that at least for one $k$

$$
\left(1-\frac{h}{2}-z^{2}-\gamma_{k}\right) J_{v}(z)-h z J_{v}^{\prime}(z)=0,
$$

where $z=\sqrt{\lambda-\gamma_{k}}$. Then, by using (2.4) and identity $z J_{v}^{\prime}(z)=z J_{v-1}(z)-v J_{v}(z)[[35]$, p. 56], we get

$$
\left(1-\frac{h}{2}-z^{2}-\gamma_{k}+h v\right) J_{v}(z)-h z J_{v-1}(z)=0,
$$

Find the eigenvalues of the operator $L_{0}$ which are less than $\gamma_{k}$. These values correspond to the imaginary roots of Equation 2.5. By taking $z=2 i \sqrt{y}$ and using [[35], p. 51]: 


$$
\sum_{n=0}^{\infty} \frac{y^{n}}{n ! \Gamma(n+v+1)}=\frac{J_{v}(2 i \sqrt{y})}{(i \sqrt{y})^{v}}
$$

we get

$$
-2 h i \sqrt{y}(i \sqrt{y})^{\nu-1} \sum_{n=0}^{\infty} \frac{y^{n}}{n ! \Gamma(n+v)}+\left(4 y+1-\frac{h}{2}+h v-\gamma_{k}\right)(i \sqrt{y})^{v} \sum_{n=0}^{\infty} \frac{\gamma^{n}}{n ! \Gamma(n+v+1)}=0,
$$

or equivalently

$$
\begin{aligned}
- & 2 h \sum_{n=0}^{\infty} \frac{y^{n}}{n ! \Gamma(n+v)}+\left(4 y+1-\frac{h}{2}-\gamma_{k}+h v\right) \sum_{n=0}^{\infty} \frac{\gamma^{n}}{n ! \Gamma(n+v+1)} \\
= & \sum_{n=0}^{\infty} \frac{\gamma^{n}}{n !}\left(\frac{-2 h+4 n}{\Gamma(n+v)}-\frac{\gamma_{k}+\frac{h}{2}-1-h v}{(v+n) \Gamma(n+v)}\right) \\
= & \sum_{n=0}^{\infty} \frac{y^{n}}{n !} \frac{(v+n)(4 n-2 h)-\left(\gamma_{k}+\frac{h}{2}-1-h v\right)}{(v+n) \Gamma(n+v)}=0 .
\end{aligned}
$$

Now, consider the quadratic equation $(4 z-2 h)(v+z)-\left(\gamma_{k}+\frac{h}{2}-1-h v\right)=0$ whose roots are given as

$$
z=\frac{-(2 v-h) \pm \sqrt{(2 v-h)^{2}+4 \gamma_{k}+2 h-4+4 h v}}{4} .
$$

Therefore, the coefficients for $y^{n}$ in (2.6) become positive for

$$
n>\left[-\frac{v-\frac{h}{2}}{2}+\frac{\sqrt{\frac{(2 v-h)^{2}}{4}+\gamma_{k}+\frac{h}{2}-1+h v}}{2}\right] .
$$

Further, let $N$ be the number of positive roots of the function in (2.6), and $W$ be the number of sign changes in its coefficients. Because the radius of convergence of this series is $\infty$, then by Descartes' rule of signs [[36], p. 52] $W-N$ is a nonnegative even number. From (2.7), $W=1$, therefore $N=1$. Hence, beginning with some $k$, Equation 2.6 has exactly one positive root corresponding to the imaginary root of Equation 2.5.

Now, find the asymptotic of the imaginary roots of Equation 2.5. For $z=i y$ and using the asymptotic of $J_{v}(z)$ for imaginary $z$ a large $|z|$ [[37], p. 976]

$$
J_{v}(i \gamma)=I_{\nu}(\gamma) e^{\frac{\pi}{2} v i}, \quad I_{\nu}(\gamma) \sim \frac{e^{\gamma}}{\sqrt{2 \pi y}}\left(1-\frac{v^{2}-\frac{1}{4}}{2 \gamma}+O\left(\frac{1}{y^{2}}\right)\right),
$$

This means (2.4) is equivalent to

$$
\left(1-\frac{h}{2}+\gamma^{2}-\gamma_{k}\right)\left(1-\frac{v^{2}-\frac{1}{4}}{2 y}+O\left(\frac{1}{\gamma^{2}}\right)\right)-h y\left(1-\frac{(v-1)^{2}-\frac{1}{4}}{2 y}+O\left(\frac{1}{\gamma^{2}}\right)\right)=0,
$$

from which

$$
y \sim \frac{v^{2}-\frac{1}{4}+2 h}{4}+\sqrt{\gamma_{k}-\frac{\sqrt{\gamma_{k}}}{2}\left(v^{2}-\frac{1}{4}\right)} .
$$


Using (2.8) in $\sqrt{\gamma_{k}-\lambda}=y$, we come up with the asymptotic formula for the eigenvalues of $L_{0}$ which are less than $\gamma_{k}$

$$
\lambda_{k} \sim-h \sqrt{\gamma_{k}} .
$$

Now, find the asymptotic of those solutions of Equation 2.3 which are greater than $\gamma_{k}$, i.e., the real roots of Equation 2.5. By virtue of the asymptotic for a large $|z|$ [[35], p. 222]

$$
J_{\nu}(z)=\sqrt{\frac{2}{\pi z}} \cos \left(z-\frac{v \pi}{2}-\frac{\pi}{4}\right)\left(1+O\left(\frac{1}{z}\right)\right),
$$

Equation 2.5 becomes

$$
\begin{gathered}
\left(1-\frac{h}{2}-z^{2}-\gamma_{k}\right) \sqrt{\frac{2}{\pi z}} \cos \left(z-\frac{v \pi}{2}-\frac{\pi}{4}\right)\left(1+O\left(\frac{1}{z}\right)\right) \\
+h z \sqrt{\frac{2}{\pi z}} \sin \left(z-\frac{v \pi}{2}-\frac{\pi}{4}\right)\left(1+O\left(\frac{1}{z}\right)\right)=0 .
\end{gathered}
$$

Hence,

$$
z=\frac{v \pi}{2}-\frac{\pi}{4}+\pi m+O\left(\frac{1}{z}\right)
$$

where $m$ is a large integer. Therefore, we can state the following Lemma 2.1:

Lemma 2.1. The eigenvalues of the operator $L_{0}$ form two sequences

$$
\lambda_{k} \sim-h \sqrt{\gamma_{k}} \text { and } \lambda_{m, k}=\gamma_{k}+z_{m}^{2}=\gamma_{k}+\alpha_{m}
$$

where $\alpha_{m} \sim\left(\pi m+\frac{v \pi}{2}-\frac{\pi}{4}\right)^{2}$. Denote the imaginary and real roots of Equation 2.2 by $x_{0, k}$ and $x_{m, k}$, respectively.

State the following two lemmas.

Lemma 2.2. Equation 2.5 has no complex roots except the pure imaginary or real roots.

Proof. $\lambda$ is real since it is eigenvalue of self-adjoint operator associated with problem (2.1), (2.2). $\gamma_{k}$ is real by our assumption $\left(A^{*}=A\right)$. Hence, the roots of $(2.5)$ are square roots of real numbers. Lemma 2.2 is proved.

Let $C$ be a rectangular contour with vertices at $\pm i B, \pm i B+A_{m}$, where $A_{m}=m \pi+\frac{v \pi}{2}+\frac{\pi}{4}$, and $B$ is a large positive number. Further, assume that this contour bypasses the origin and the imaginary root at $-i x_{0, k}$ along the small semicircle on the right side of the imaginary axis and $i x_{0, k}$ on the left.

Then, we claim that the following lemma is true.

Lemma 2.3. For a sufficiently large integer $m$, the number of zeros of the function

$$
z^{-v}\left(\left(1-\frac{h}{2}-z^{2}-\gamma_{k}\right) J_{v}(z)-h z J^{\prime}{ }_{v}(z)\right)
$$

inside of $C$ is exactly $m$.

Proof. Since $z^{-v}\left(\left(1-\frac{h}{2}-z^{2}-\gamma_{k}\right) J_{v}(z)-h z J^{\prime}{ }_{v}(z)\right)$ is an entire function of $z$, then the number of its zeros inside of $C$ equals: 


$$
\begin{aligned}
& \frac{1}{2 \pi i} \int_{C} \frac{\left[z^{-v}\left(\left(1-\frac{h}{2}-z^{2}-\gamma_{k}\right) J_{v}(z)-h z J_{v}^{\prime}(z)\right)\right]^{\prime}}{z^{-v}\left(\left(1-\frac{h}{2}-z^{2}-\gamma_{k}\right) J_{v}(z)-h z J_{v}^{\prime}(z)\right)} d z \\
& =\frac{1}{2 \pi i} \int_{C} \frac{\left[z^{-v}\left(h z J_{v+1}(z)+\left(1-\frac{h}{2}-h v-z^{2}-\gamma_{k}\right) J_{v}(z)\right)\right]^{\prime}}{z^{-v}\left(\left(1-\frac{h}{2}-z^{2}-\gamma_{k}\right) J_{v}(z)-h z J_{v}^{\prime}(z)\right)} d z \\
& =\frac{1}{2 \pi i} \int_{C}\left[\frac{-v z^{-\nu-1}\left(h z J_{v+1}(z)+\left(1-\frac{h}{2}-h v-z^{2}-\gamma_{k}\right) J_{v}(z)\right)}{z^{-v}\left(\left(1-\frac{h}{2}-z^{2}-\gamma_{k}\right) J_{v}(z)-h z J_{v}^{\prime}(z)\right)}\right. \\
& \left.+\frac{z^{-v}\left(h J_{v+1}(z)+h z J_{v+1}^{\prime}(z)-2 z J_{v}(z)+\left(1-\frac{h}{2}-h v-z^{2}-\gamma_{k}\right) J_{v}^{\prime}(z)\right)}{z^{-v}\left(\left(1-\frac{h}{2}-z^{2}-\gamma_{k}\right) J_{v}(z)-h z J_{v}^{\prime}(z)\right)}\right] d z \\
& =\frac{1}{2 \pi i} \int_{C}\left[\frac{z^{-v}\left(-v h J_{v+1}(z)-v \frac{J_{v}(z)}{z}\left(1-\frac{h}{2}-h v-z^{2}-\gamma_{k}\right)\right)}{z^{-v}\left(\left(1-\frac{h}{2}-z^{2}-\gamma_{k}\right) J_{v}(z)-h z J_{v}^{\prime}(z)\right)}\right. \\
& \left.+\frac{z^{-v}\left(h J_{v+1}(z)+h z J_{v+1}^{\prime}(z)-2 z J_{v}(z)+\left(1-\frac{h}{2}-h v-z^{2}-\gamma_{k}\right) J_{v}^{\prime}(z)\right)}{z^{-v}\left(\left(1-\frac{h}{2}-z^{2}-\gamma_{k}\right) J_{v}(z)-h z J_{v}^{\prime}(z)\right)}\right] d z \\
& =\frac{1}{2 \pi i} \int_{C}\left[\frac{z^{-\nu}\left(-v h J_{v+1}(z)-J_{\nu+1}(z)\left(1-\frac{h}{2}-h v-z^{2}-\gamma_{k}\right)\right.}{z^{-v}\left(\left(1-\frac{h}{2}-z^{2}-\gamma_{k}\right) J_{\nu}(z)-h z J_{\nu}^{\prime}(z)\right)}\right. \\
& \left.+\frac{z^{-v}\left(h J_{v+1}(z)+h z J_{v}(z)-h(v+1) J_{v+1}(z)-2 z J_{v}(z)\right)}{z^{-v}\left(\left(1-\frac{h}{2}-z^{2}-\gamma_{k}\right) J_{v}(z)-h z J_{v}^{\prime}(z)\right)}\right] d z \\
& =\frac{1}{2 \pi i} \int_{C} \frac{-J_{v+1}(z)\left(1-\frac{h}{2}-z^{2}-\gamma_{k}\right)-2 v h J_{v+1}(z)+J_{v}(z) z(h-2)}{\left(1-\frac{h}{2}-z^{2}-\gamma_{k}\right) J_{v}(z)-h z J_{v}^{\prime}(z)} d z .
\end{aligned}
$$

In the above, we have used the following identities:

$$
\begin{gathered}
z J^{\prime}{ }_{v}(z)=v J_{v}(z)-z J_{v+1}(z), \\
z J^{\prime}{ }_{v+1}(z)=z J_{v}(z)-(v+1) J_{v+1}(z) .
\end{gathered}
$$

As the integrand is an odd function. the order of its numerator in the vicinity of zero is $O\left(z^{v+1}\right)$, and the order of its denominator is $O\left(z^{v}\right)$, the integral along the left part of contour vanishes. Now, consider the integrals along the remaining three sides of the contour. On these sides [[35], p. 221, p. 88]

$$
J_{v}(z)=\frac{H_{v}^{(1)}(z)+H_{v}^{(2)}(z)}{2}
$$

where

$$
\begin{aligned}
& H_{v}^{(1)}(z)=\left(\frac{2}{\pi z}\right)^{\frac{1}{2}} e^{i\left(z-\frac{v \pi}{2}-\frac{\pi}{4}\right)}\left\{1+\eta_{1, v}(z)\right\}, \\
& H_{v}^{(2)}(z)=\left(\frac{2}{\pi z}\right)^{\frac{1}{2}} e^{-i\left(z-\frac{v \pi}{2}-\frac{\pi}{4}\right)}\left\{1+\eta_{2, v}(z)\right\}
\end{aligned}
$$

$\eta_{1, v}(z)$ and $\eta_{2, v}(z)$ are of order $O\left(\frac{1}{z}\right)$ for large $|z|$. 
For simplicity, denote the integrand by $f(z)$, then

$$
\begin{aligned}
& \frac{1}{2 \pi i} \int_{i B+m \pi+\frac{v \pi}{2}+\frac{\pi}{4}}^{i B} f(z) d z=-\frac{1}{2 \pi i} \int_{i B}^{i B+m \pi+\frac{v \pi}{2}+\frac{\pi}{4}} f(z) d z \\
& \quad \sim \frac{1}{2 \pi i} \int_{i B}^{i B+m \pi+\frac{v \pi}{2}+\frac{\pi}{4}} \frac{J_{v+1}(z)}{J_{v}(z)}\left(1+O\left(\frac{1}{z}\right)\right) d z \\
& \quad=\frac{1}{2 \pi i} \int_{i B}^{i B+m \pi+\frac{v \pi}{2}+\frac{\pi}{4}}\left[\frac{1+\eta_{2, v+1}(z)}{1+\eta_{2, v}(z)}\right]\left[1+O\left(e^{2 i z}\right)\right] d z \rightarrow \frac{m}{2}+\frac{v}{4}+\frac{1}{8} .
\end{aligned}
$$

One can analogously show that the integral along the lower side tends to the same number.

To calculate the integral along the fourth side, use the relations: $\frac{J_{v+1}(z)}{J_{\nu}(z)}-\operatorname{tg}\left(z-\frac{v \pi}{2}-\frac{\pi}{4}\right)=\frac{2 v+1}{2 z}+O\left(\frac{1}{z^{2}}\right) \quad$ for $\quad$ large $\quad|z|, \quad$ and $\int_{-i B+m \pi+\frac{v \pi}{2}+\frac{\pi}{4}}^{i B+m \pi+\frac{v \pi}{4}} \operatorname{tg}\left(z-\frac{v \pi}{2}-\frac{\pi}{4}\right) d z=0$.

Since $O\left(\frac{1}{z}\right)$ is bounded on the right-hand side of the contour, we get

$$
\begin{aligned}
& -\frac{1}{2 \pi i} \int_{-i B+m \pi+\frac{v \pi}{2}+\frac{\pi}{4}}^{i B+m \pi+\frac{v \pi}{2}+\frac{\pi}{4}}\left[\frac{J_{v+1}(z)}{J_{v}(z)}\left(1+O\left(\frac{1}{z}\right)\right)\right] d z \\
& =-\frac{1}{2 \pi i} \int_{-i B+m \pi+\frac{v \pi}{2}+\frac{\pi}{4}}^{i B+m \pi+\frac{v \pi}{2}+\frac{\pi}{4}}\left[\frac{2 v+1}{2 z}+\operatorname{tg}\left(z-\frac{v \pi}{2}-\frac{\pi}{4}\right)+O\left(\frac{1}{z^{2}}\right)\right] \\
& \quad \times\left(1+O\left(\frac{1}{z}\right)\right) d z \sim-\frac{1}{4}(2 v+1)+O\left(\frac{1}{m}\right) .
\end{aligned}
$$

Consequently, the limit of the integral along the entire contour is $m+O\left(\frac{1}{m}\right)$. However, as the integral must be an integer, it should be equal to $m$. This completes the proof of Lemma 2.3.

By using the above results, derive the asymptotic formula for the eigenvalue distribution of $L_{0}$. To do that, denote the eigenvalue distribution of the operator $L_{0}$ by $N(\lambda$, $\left.L_{0}\right)$. Then:

$$
N\left(\lambda, L_{0}\right)=\sum_{\lambda_{j}\left(L_{0}\right)<\lambda} 1=N_{1}(\lambda)+N_{2}(\lambda),
$$

where

$$
N_{1}(\lambda)=\sum_{\lambda_{k}<\lambda} 1, \quad N_{2}(\lambda)=\sum_{\lambda_{m, k}<\lambda} 1 .
$$

Since $\gamma_{k} \sim a \cdot k^{\alpha}$, then $\lambda_{k} \sim c_{1} k^{\frac{\alpha}{2}}$. That is

$$
N_{1}(\lambda) \sim C_{1} \lambda^{\frac{2}{\alpha}} .
$$


From Lemmas 2.2 and 2.3 and the asymptotic of $x_{m, k}$, it follows that one can find a number $c$ such that for a large $m$

$$
\pi m<x_{m, k}<\pi m+c .
$$

From this inequality, it follows that $N_{2}(\lambda)$ is less than $N_{2}^{\prime}(\lambda)$, where $N_{2}^{\prime}(\lambda)$ is the number of the positive integer pairs $(m, k)$ satisfying the inequality

$$
\pi^{2} m^{2}+a k^{\alpha}<\lambda .
$$

Also, $N_{2}(\lambda)$ is greater than $N_{2}^{\prime \prime}(\lambda)$, where $N_{2}^{\prime \prime}(\lambda)$ is the number of the positive integer pairs for which

$$
(\pi m+c)^{2}+a k^{\alpha}<\lambda .
$$

To summarize, we have

$$
N_{2}^{\prime \prime}(\lambda)<N_{2}(\lambda) \leq N_{2}^{\prime}(\lambda) .
$$

Thus, by (2.12) and (2.13) as in [[38], Section 3, Lemma 2] we have:

$$
\frac{2 \gamma \lambda^{\frac{2+\alpha}{2 \alpha}}}{\pi \alpha \sqrt{a}}-(c+1)\left(\frac{\lambda}{a}\right)^{\frac{1}{\alpha}}-\frac{1}{\pi} \lambda^{\frac{1}{2}} \leq N_{2}^{\prime \prime}(\lambda)<N_{2}(\lambda) \leq N_{2}^{\prime}(\lambda) \leq \frac{2 \gamma \lambda^{\frac{2+\alpha}{2 \alpha}}}{\pi \alpha \sqrt{a}}
$$

where $\gamma=\int_{0}^{\pi} \cos ^{2} t \sin ^{\frac{2}{\alpha}-1} t d t$

From the above, we have

$$
N_{2}(\lambda) \sim \frac{2 \gamma \lambda^{\frac{2+\alpha}{2 \alpha}}}{\pi \alpha \sqrt{a}}
$$

Therefore, by virtue of (2.11) and (2.15), we have

$$
N(\lambda) \sim c_{1} \lambda^{\frac{2}{\alpha}}+c_{2} \lambda^{\frac{2+\alpha}{2 \alpha}}
$$

For $\alpha>2$

$$
N\left(\lambda, L_{0}\right) \sim c_{2} \lambda^{\frac{2+\alpha}{2 \alpha}}
$$

and consequently, $\lambda_{n}\left(L_{0}\right) \sim d n^{\frac{2+\alpha}{2 \alpha}}, d=c_{2}^{-\frac{2 \alpha}{2+\alpha}}$.

For $\alpha>2, N\left(\lambda, L_{0}\right) \sim c_{1} \lambda^{\frac{2}{\alpha}}$ or, $\lambda_{n}\left(L_{0}\right) \sim c_{1}^{-\frac{2}{\alpha}} n^{\frac{\alpha}{2}}$.

For $\alpha=2, N(\lambda) \sim\left(c_{1}+c_{2}\right) \lambda$ from which $\lambda_{n}\left(L_{0}\right) \sim d n, d=\left(c_{1}+c_{2}\right)^{-1}$.

Then, as $Q$ is a bounded operator in $\mathbf{L}_{2}$, it follows from the relation for the resolvents of the operators $L_{0}$ and $L$ [[30], p. 219]

$$
R_{\lambda}(L)=R_{\lambda}\left(L_{0}\right)-R_{\lambda}(L) Q R_{\lambda}\left(L_{0}\right)
$$

that the spectrum of $L$ is also discrete. By virtue of the last equality and the properties that hold for $s$ numbers of compact operators [[30], pp. 44, 49] as in [[38], Section 3 , Lemma 2], for the eigenvalues of $\mathrm{L}$ denoted by $\mu_{n}(L)$, we have

$$
\mu_{n}(L) \sim d n^{\delta} .
$$


Therefore, we can state the following theorem:

Theorem 2.1. If $\gamma_{n} \sim a n^{\alpha}(0<a, \alpha>0)$, then

$$
\lambda_{n}\left(L_{0}\right) \sim \mu_{n}(L) \sim d n^{\delta},
$$

where

$$
\delta= \begin{cases}\frac{2 \alpha}{\alpha+2} & \text { for } \alpha>2, \\ \frac{\alpha}{2} & \text { for } \alpha<2, \\ 1 & \text { for } \alpha=1 .\end{cases}
$$

For simplicity, we will denote the eigenvalues of $L_{0}$ and $L$ by $\lambda_{n}$ and $\mu_{n}$, respectively.

\section{Regularized trace of the operator $L$}

Now make use of the theorem proved in [20] for abstract operators. At first, introduce the following notations.

Let $A_{0}$ be a self-adjoint positive discrete operator, $\left\{\lambda_{n}\right\}$ be its eigenvalues arranged in ascending order, $\left\{\phi_{j}\right\}$ be a basis formed by the eigenvectors of $A_{0}, B$ be a perturbation operator, and $\left\{\mu_{n}\right\}$ be the eigenvalues of $A_{0}+B$. Also, assume that $A_{0}^{-1} \in \sigma_{1}(H)$. For operators $A_{0}$ and $B$ in [[20], Theorem 1], the following theorem is proved.

Theorem 3.1. Let the operator $B$ be such that $D\left(A_{0}\right) \subset D(B)$, and let there exist a number $\delta \in[0,1)$ such that $B A_{0}^{-\delta}$ has a bounded extension, and number $\omega \in[0,1), \omega$ $+\delta<1$ such that $A_{0}^{-(1-\delta-\omega)}$ is a trace class operator. Then, there exists a subsequence of natural numbers $\left\{n_{m}\right\}_{m=1}^{\infty}$ and a subsequence of contours $\Gamma_{m} \in C$, that for $\omega \geq \delta$ the formula

$$
\lim _{m \rightarrow \infty} \sum_{j=1}^{n_{m}}\left(\mu_{j}-\lambda_{j}-\left(B \varphi_{j}, \varphi_{j}\right)\right)=0
$$

is true.

Note that the conditions of this theorem are satisfied for $L_{0}$ and $L$. That is, if we take $A_{0}=L_{0}, B=Q$, then $L_{0}^{-1} Q$ is bounded. For $\omega=\delta<\frac{\alpha-2}{4 \alpha}$ and $\alpha>2$, from asymptotic (2.16), we will have that $A_{0}^{-(1-\delta-\omega)}=L_{0}^{-(1-2 \delta)}$ is a trace class operator. If $\alpha<2$, then $L_{0}^{-(1-2 \delta)}$ will be a trace class operator for $\omega=\delta<\frac{\alpha-2}{\alpha}$.

Thus, by the statement of Theorem 3.1, for $\alpha>2$, we have

$$
\lim _{m \rightarrow \infty} \sum_{n=1}^{n_{m}}\left(\mu_{n}-\lambda_{n}\right)=\lim _{m \rightarrow \infty} \sum_{n=1}^{n_{m}}\left(Q \psi_{n}, \psi_{n}\right)_{\mathbf{L}_{2}},
$$

where $\psi_{1}(x), \psi_{2}(x), \ldots$ are the orthonormal eigenvectors of $L_{0}$. Introduce the following notation:

$$
\lambda^{(i)}=\sum_{k=n_{i-1}+1}^{n_{i}} \lambda_{k}, \quad \mu^{(i)}=\sum_{k=n_{i-1}+1}^{n_{i}} \mu_{k} \quad(i=1,2, \ldots),
$$

and investigate the sum of series $\sum_{i=1}^{\infty}\left(\mu^{(i)}-\lambda^{(i)}\right)$, which as will be seen later, is independent of the choice of $\left\{n_{m}\right\}_{m=1}^{\infty}$. We will call the sum of this series a regularized trace of the operator $L_{0}$. 
Now, we calculate the norm for the eigen-vectors of the operator $L_{0}$ in $\mathbf{L}_{2}$. To do this, we will use the following identity obtained from the Bessel equation"

$$
\int_{0}^{1} t J_{v}(\alpha t) J_{v}(\beta t) d t=\frac{1}{\alpha^{2}-\beta^{2}}\left[J_{v}(\alpha) \beta J^{\prime}{ }_{v}(\beta)-J_{v}(\beta) \alpha J^{\prime}{ }_{v}(\alpha)\right] .
$$

As $\alpha \rightarrow \beta$, we get

$$
\int_{0}^{1} t J_{v}^{2}(\beta t) d t=\frac{\beta^{2} J^{\prime}{ }_{v}(\beta)^{2}+\left(\beta^{2}-v^{2}\right) J_{v}^{2}(\beta)}{2 \beta^{2}} .
$$

We also consider the following identities:

$$
\begin{gathered}
J^{\prime}{ }_{v}(z)=-J_{v+1}(z)+\frac{v}{z} J_{v}(z) \\
J^{\prime \prime}{ }_{v}(z)=-J^{\prime}{ }_{v+1}(z)-\frac{v}{z^{2}} J_{v}(z)+\frac{v}{z} J^{\prime}{ }_{v}(z), \\
z J^{\prime}{ }_{v+1}(z)=z J_{v}(z)-(v+1) J_{v+1}(z) .
\end{gathered}
$$

By the above identities and also by the equation

$$
\beta J_{v}^{\prime}(\beta)-\left(1-\frac{h}{2}-\beta^{2}-\gamma_{k}\right) \frac{J_{v}(\beta)}{h}=0
$$

satisfied by $x_{m, k}$, we obtain

$$
\begin{aligned}
& \left(\beta^{2}-v^{2}\right) J_{v}^{2}(\beta)+\beta^{2} J_{v}^{\prime 2}(\beta) \\
& \quad=\frac{1-h-2 \beta^{2}-2 \gamma_{k}+\frac{h^{2}}{4}+\beta^{2} h+\gamma_{k} h+\beta^{4}+2 \gamma_{k} \beta^{2}+\gamma_{k}^{2}+\beta^{2} h^{2}-v^{2} h^{2}}{h^{2}} J_{v}^{2}(\beta) .
\end{aligned}
$$

Therefore,

$$
\begin{aligned}
& \int_{0}^{1} t J_{v}^{2}\left(x_{m, k} t\right)\left(\varphi_{k}, \varphi_{k}\right) d t-\frac{1}{h} J_{v}^{2}\left(x_{m, k}\right)\left(\varphi_{k}, \varphi_{k}\right) \\
& \quad=\frac{1-h-2 x_{m, k}^{2}-2 \gamma_{k}+\frac{h^{2}}{4}-x_{m, k}^{2} h+\gamma_{k} h+x_{m, k}^{4}+2 \gamma_{k} x_{m, k}^{2}+\gamma_{k}^{2}+h^{2} x_{m, k}^{2}-v^{2} h^{2}}{2 x_{m, k}^{2} h^{2}} J_{v}^{2}\left(x_{m, k}\right) .
\end{aligned}
$$

So, the orthonormal eigen-vectors of $L_{0}$ are

$$
\begin{aligned}
& \frac{1}{J_{v}\left(x_{m, k}\right)} \sqrt{\frac{2 x_{m, k}^{2} h^{2}}{1-h-2 x_{m, k}^{2}-2 \gamma_{k}+\frac{h^{2}}{4}-x_{m, k}^{2}+\gamma_{k} h+x_{m, k}^{4}+2 \gamma_{k} x_{m, k}^{2}+\gamma_{k}^{2}+h^{2} x_{m, k}^{2}-v^{2} h^{2}}} \\
& \quad \times\left\{\sqrt{t} J_{v}\left(x_{m, k} t\right) \varphi_{k} J_{v}\left(x_{m, k}\right) \varphi_{k}\right\} \quad\left(\begin{array}{lr}
m=\overline{1, \infty} ; & k=\overline{1, \infty} \\
m=0 ; \quad & k=\overline{N, \infty}
\end{array}\right) .
\end{aligned}
$$

Now, we prove the following lemma.

Lemma 3.1. If the operator function $q(t)$ has properties 1,2 , and also $\alpha>0$, then

$$
\begin{gathered}
\sum_{k=1}^{\infty} \sum_{m=1}^{\infty}\left|\int_{0}^{1} \frac{2 x_{m, k}^{2} h^{2} t J_{v}^{2}\left(x_{m, k} t\right)\left(q(t) \varphi_{k}, \varphi_{k}\right) d t}{J_{v}^{2}\left(x_{m, k}\right)\left(1-h-2 x_{m, k}^{2}-2 \gamma_{k}+\frac{h^{2}}{4}-x_{m, k}^{2} h+\gamma_{k} h+x_{m, k}^{4}+2 \gamma_{k} x_{m, k}^{2}+\gamma_{k}^{2}+h^{2} x_{m, k}^{2}-v^{2} h^{2}\right)}\right| \\
+\sum_{k=N}^{\infty}\left|\int_{0}^{1} \frac{2 x_{0, k}^{2} h^{2} t J_{v}^{2}\left(x_{0,} k^{t}\right)\left(q(t) \varphi_{k}, \varphi_{k}\right) d t}{J_{v}^{2}\left(x_{0, k}\right)\left(1-h-2 x_{0, k}^{2}-2 \gamma_{k}+\frac{h^{2}}{4}-x_{0, k}^{2} h+\gamma_{k} h+x_{0, k}^{4}+2 \gamma_{k} x_{0, k}^{2}+\gamma_{k}^{2}+h^{2} x_{0, k}^{2}-v^{2} h^{2}\right)}\right|<\infty .
\end{gathered}
$$


Proof. Assume that $f_{k}(t)=\left(q(t) \phi_{k}, \phi_{k}\right)$. By Lemma 2.1 we have $x_{m, k} \sim \pi m+\frac{v \pi}{2}-\frac{\pi}{4}$. So, in virtue of the inequality $\left|\frac{t t_{v}^{2}\left(x_{m, k} t\right)}{J_{v}^{2}\left(x_{m, k}\right)}\right|<c[[35]$, p. 666] and properties 1 and 2 we have

$$
\begin{aligned}
& \sum_{k=1}^{\infty} \sum_{m=1}^{\infty}\left|\int_{0}^{1} \frac{2 x_{m, k}^{2} h^{2} t t_{v}^{2}\left(x_{m, k} t\right) f_{k}(t) d t}{J_{v}^{2}\left(x_{m, k}\right)\left(1-h-2 x_{m, k}^{2}-2 \gamma_{k}+\frac{h^{2}}{4}-x_{m, k}^{2} h+\gamma_{k} h+x_{m, k}^{4}+2 \gamma_{k} x_{m, k}^{2}+\gamma_{k}^{2}+h^{2} x_{m, k}^{2}-v^{2} h^{2}\right)}\right| \\
& \quad<c \sum_{k=1}^{\infty} \sum_{m=1}^{\infty} \int_{0}^{1} \frac{\left|f_{k}(t)\right| d t}{x_{m, k}^{2}-h+2 \gamma_{k}+h^{2}-2+\frac{1-h-2 \gamma_{k}+\frac{h^{2}}{4}+\gamma_{k} h+\gamma_{k}^{2}-v^{2} h^{2}}{x_{m, k}^{2}}} \\
& \quad \sim \sum_{k=1}^{\infty} \sum_{m=1}^{\infty} O\left(\frac{1}{x_{m, k}^{2}}\right) \int_{0}^{1}\left|f_{k}(t)\right| d t<\infty .
\end{aligned}
$$

To estimate the second series in (3.5), we use the relation $x_{0, k} \sim \frac{v^{2}-\frac{1}{4}+2 h}{4}+\sqrt{\gamma_{k}}, \gamma_{k} \sim a k^{\alpha}$.

By hypothesis of Lemma $3.1 \alpha>0$. Therefore, denoting this sum by $s$, we have

$$
|s|<\sum_{k=N}^{\infty} \frac{1}{x_{0, k}^{2}} \int_{0}^{1}\left|f_{k}(t) d t\right|<\infty .
$$

This proves Lemma 3.1.

Now, assume that

$$
\begin{aligned}
& \int_{1-\delta}^{1} \frac{\left|f_{k}(t)\right|}{\cos \frac{\pi t}{2}} d t<\infty, \\
& \int_{0}^{\delta} \frac{\left|f_{k}(t)\right|}{t} d t<\infty
\end{aligned}
$$

for small $\delta>0$.

Then, we can state the following theorem.

Theorem 3.2. Let the conditions of Theorem 2.1, (3.6) and (3.7) hold. If the operatorvalue function $q(t)$ has properties 1-3, then the following formula is true

$$
\lim _{m \rightarrow \infty} \sum_{i=1}^{n_{m}}\left(\mu_{n}-\lambda_{n}\right)=-\frac{2 \nu \operatorname{trq}(0)+\operatorname{trq}(1)}{4} .
$$

Proof. By virtue of lemma 3.1 we have

$$
\begin{gathered}
\sum_{i=1}^{\infty}\left(\mu^{(i)}-\lambda^{(i)}\right) \\
=\sum_{k=1}^{N-1} \sum_{m=1}^{\infty} \int_{0}^{1} \frac{2 x_{m, k}^{2} h^{2} t J_{v}^{2}\left(x_{m, k} t\right) f_{k}(t)}{\left[1-h-2 x_{m, k}^{2}-2 \gamma_{k}+\frac{h^{2}}{4}-x_{m, k}^{2} h+\gamma_{k} h+x_{m, k}^{4}+2 \gamma_{k} x_{m, k}^{2}+\gamma_{k}^{2}+h^{2} x_{m, k}^{2}-v^{2} h^{2}\right] J_{v}^{2}\left(x_{m, k}\right)} d t \\
+\sum_{k=N}^{\infty} \sum_{m=0}^{\infty} \int_{0}^{1} \frac{2 x_{m, k}^{2} h^{2} t J_{v}^{2}\left(x_{m, k} t\right) f_{k}(t)}{\left[1-h-2 x_{m, k}^{2}-2 \gamma_{k}+\frac{h^{2}}{4}-x_{m, k}^{2} h+\gamma_{k} h+x_{m, k}^{4}+2 \gamma_{k} x_{m, k}^{2}+\gamma_{k}^{2}+h^{2} x_{m, k}^{2}-v^{2} h^{2}\right] J_{v}^{2}\left(x_{m, k}\right)} d t .
\end{gathered}
$$

At first evaluate the inner sum in the second term on the right hand side of (3.9). To do this, as $N \rightarrow \infty$ investigate the asymptotic behavior of the function 


$$
R_{N}(t)=\sum_{m=0}^{N-1} \frac{2 t h^{2} x_{m, k}^{2} J_{v}^{2}\left(x_{m, k} t\right)}{J_{v}^{2}\left(x_{m, k}\right)\left\{1-h-2 x_{m, k}^{2}-2 \gamma_{k}+\frac{h^{2}}{4}-x_{m, k}^{2} h+\gamma_{k} h+x_{m, k}^{4}+2 \gamma_{k} x_{m, k}^{2}+\gamma_{k}^{2}+h^{2} x_{m, k}^{2}-v^{2} h^{2}\right\}}
$$

To derive a formula for $R_{N}(t)$, show for each fixed value of $k$, the $m$ th term of the sum $R_{N}(t)$ as a residue at the point $x_{m, k}$ of some complex variable function with poles at $x_{m, k}(m=\overline{0, N-1})$.

For this purpose, consider the following function:

$$
g(z)=\frac{2 t z h J_{v}^{2}(t z)}{J_{v}(z)\left\{-h z J^{\prime}{ }_{v}(z)+\left(1-\frac{h}{2}-z^{2}-\gamma_{k}\right) J_{v}(z)\right\}} .
$$

The poles of this function are $x_{0, k, \ldots,}, x_{N-1, k}$ and $j_{1}, \ldots, j_{N}\left(J_{v}\left(j_{n}\right)=0\right)$. The residue at $j_{n}$ equals

$$
\operatorname{res}_{z=j_{n}} g(z)=\frac{2 t h j_{n} J_{v}^{2}\left(t j_{n}\right)}{J^{\prime}{ }_{\nu}\left(j_{n}\right)\left(-h j_{n} J^{\prime}{ }_{\nu}\left(j_{n}\right)+\left(1-\frac{h}{2}-j_{n}^{2}-\gamma_{k}\right) J_{v}\left(j_{n}\right)\right)}=-\frac{2 t J_{\nu}^{2}\left(t j_{n}\right)}{J^{\prime}{ }_{\nu}\left(j_{n}\right)^{2}} .
$$

Now, compute the residue at $x_{m, k}$ :

$$
\begin{aligned}
& \left(-h z J^{\prime}{ }_{\nu}(z)+\left(1-\frac{h}{2}-z^{2}-\gamma_{k}\right) J_{v}(z)\right)^{\prime} \\
& =J^{\prime}{ }_{v}(z)\left(1-\frac{h}{2}-z^{2}-\gamma_{k}-h\right)-h z J^{\prime \prime}{ }_{\nu}(z)-2 z J_{v}(z) \\
& =\left(-\frac{v}{z} J_{v}(z)+J_{v-1}(z)\right)\left(1-\frac{h}{2}-z^{2}-\gamma_{k}-h\right)-h z\left(\frac{v}{z^{2}} J_{v}(z)-\frac{v}{z} J^{\prime}{ }_{v}(z)+J^{\prime}{ }_{v-1}(z)\right)-2 z J_{v}(z) \\
& =-\frac{v}{z} J_{v}(z)\left(1-\frac{h}{2}-z^{2}-\gamma_{k}\right)+h v J^{\prime}{ }_{v}(z)+J_{v-1}(z)\left(1-\frac{h}{2}-z^{2}-\gamma_{k}-h\right)-h z J^{\prime}{ }_{v-1}(z)-2 z J_{v}(z) .
\end{aligned}
$$

Denote the right hand side of (3.10) by $G(z)$. Since $x_{m, k}$ satisfies equation (2.4), by setting $z=x_{m, k}$ and using the identity

$$
z J_{v-1}^{\prime}(z)=(v-1) J_{v-1}(z)-z J_{v}(z)
$$

we have

$$
\begin{aligned}
& G\left(x_{m, k}\right)=J_{v-1}\left(x_{m, k}\right)\left(1-\frac{3 h}{2}-x_{m, k}^{2}-\gamma_{k}\right)-h\left((v-1) J_{\nu-1}\left(x_{m, k}\right)-x_{m, k} J_{v}\left(x_{m, k}\right)\right) \\
& -2 x_{m, k} J_{v}\left(x_{m, k}\right)=J_{v-1}\left(x_{m, k}\right)\left(1-\frac{3 h}{2}-x_{m, k}^{2}-\gamma_{k}\right) \\
& -h(v-1) J_{v-1}\left(x_{m, k}\right)+x_{m, k}(h-2) J_{v}\left(x_{m, k}\right) \\
& =J_{v-1}\left(x_{m, k}\right)\left(1-\frac{h}{2}-x_{m, k}^{2}-\gamma_{k}-h v\right)+(h-2) x_{m, k} J_{v}\left(x_{m, k}\right) \\
& =\left(J_{\nu}^{\prime}\left(x_{m, k}\right)+\frac{v}{x_{m, k}} J_{v}\left(x_{m, k}\right)\right)\left(1-\frac{h}{2}-x_{m, k}^{2}-\gamma_{k}-h v\right)+(h-2) x_{m, k} J_{v}\left(x_{m, k}\right) \\
& =\frac{\left(x_{m, k} J_{\nu}^{\prime}\left(x_{m, k}\right)+v J_{v}\left(x_{m, k}\right)\right)\left(1-h v-\frac{h}{2}-x_{m, k}^{2}-\gamma_{k}\right)+(h-2) x_{m, k}^{2} J_{v}^{2}\left(x_{m, k}\right)}{x_{m, k}} \\
& =\frac{-h v x_{m, k} J^{\prime}{ }_{\nu}\left(x_{m, k}\right)-h \nu^{2} J_{\nu}\left(x_{m, k}\right)}{x_{m, k}}+\frac{\left(x_{m, k} J^{\prime}{ }_{\nu}\left(x_{m, k}\right)+v J_{v}\left(x_{m, k}\right)\right)\left(1-\frac{h}{2}-x_{m, k}^{2}-\gamma_{k}\right)+(h-2) x_{m, k}^{2} J_{\nu}^{2}\left(x_{m, k}\right)}{x_{m, k}} \\
& =\frac{-h v^{2} J_{v}\left(x_{m, k}\right)+x_{m, k} J^{\prime}{ }_{\nu}\left(x_{m, k}\right)\left(1-\frac{h}{2}-x_{m, k}^{2}-\gamma_{k}\right)+(h-2) x_{m, k}^{2} J_{v}\left(x_{m, k}\right)}{x_{m, k}} \\
& =\frac{J_{v}\left(x_{m, k}\right)\left[\left(-h v^{2}+(h-2) x_{m, k}^{2}\right)+\frac{\left(1-\frac{h}{2}-x_{m, k}^{2}-\gamma_{k}\right)^{2}}{h}\right]}{x_{m, k}}=\frac{J_{v}\left(x_{m, k}\right)}{x_{m, k}} \\
& \times \frac{\left[1-h-2 x_{m, k}^{2}-2 \gamma_{k}+\frac{h^{2}}{4}-x_{m, k}^{2} h+\gamma_{k} h+x_{m, k}^{4}+2 \gamma_{k} x_{m, k}^{2}+\gamma_{k}^{2}-h^{2} v^{2}+h^{2} x_{m, k}^{2}\right]}{h} .
\end{aligned}
$$


Therefore,

$$
\operatorname{res}_{z=x_{m, k}} g(z)=\frac{2 t h^{2} x_{m, k}^{2} J_{v}^{2}\left(t x_{m, k}\right) / J_{v}^{2}\left(x_{m, k}\right)}{\left(1-h-2 x_{m, k}^{2}-2 \gamma_{k}+\frac{h^{2}}{4}-x_{m, k}^{2} h+\gamma_{k} h+x_{m, k}^{4}+2 \gamma_{k} x_{m, k}^{2}+\gamma_{k}^{2}+h^{2} x_{m, k}^{2}-v^{2} h^{2}\right)} .
$$

Consider the contour $C$ mentioned in Lemma 2.3 as the contour of integration. According to Lemmas 2.1 and 2.3, for a sufficiently large $N$, we have $x_{N-1, k}<A_{N}<x_{N}$, $k$ and $j_{N}<A_{N}<j_{N+1}$.

It could easily be verified that in the vicinity of zero, the function $g(z)$ is of order $O\left(z^{v}\right)$. By virtue of this asymptotic and because $g(z)$ is an odd function, the integral along the left-hand side of the contour $C$ vanishes when $r$ (radius of a semicircle) goes to zero.

Furthermore, if $z=u+i v$, then for large $|v|$ and $u \geq 0$, the integrand will be of order $O\left(e^{|v|(2 t-2)}\right)$. That is, for a given value of $A_{N}$, the integrals along the upper and lower sides of $C$ go to zero as $B \rightarrow \infty(0<t<1)$. Thus, we obtain

$$
R_{N}(t)-T_{N}(t)=\lim _{B \rightarrow \infty} \frac{1}{2 \pi i} \int_{A_{N}-i B}^{A_{N}+i B} \frac{2 z t h J_{\nu}^{2}(t z) d z}{J_{v}(z)\left\{-h z J^{\prime}{ }_{\nu}(z)+\left(1-\frac{h}{2}-z^{2}-\gamma_{k}\right) J_{v}(z)\right\}},
$$

where

$$
T_{N}(t)=\sum_{n=1}^{N} \frac{2 t J_{v}^{2}\left(t j_{h}\right)}{J^{\prime}{ }_{v}\left(j_{h}\right)^{2}}
$$

Also, along the contour $C$ for $x_{N-1, k}^{-1+\varepsilon} \leq t<1,0<\varepsilon<\frac{1}{2}$, we have $|t z| \rightarrow \infty$. Therefore, in integral (3.11), we could replace the Bessel functions by their asymptotic at large arguments. Hence, from

$$
J_{v}^{2}(z)=\frac{2}{\pi z}\left[\frac{1}{2}+\frac{\sin (2 z-v \pi)}{2}\right]\left(1+O\left(\frac{1}{z}\right)\right)
$$

as $N \rightarrow \infty$ we have

$$
\begin{aligned}
& \frac{1}{2 \pi i} \lim _{B \rightarrow \infty} \int_{A_{N}-i B}^{A_{N}+i B} g(z) d z \sim \frac{h}{\pi i} \lim _{B \rightarrow \infty} \int_{A_{N}-i B}^{A_{N}+i B} \frac{1+\sin (2 z t-v \pi)}{-z(1+\sin (2 z-v \pi))} d z \\
& \sim \frac{1}{\pi} \int_{-\infty}^{\infty} \frac{d v}{-\left(A_{N}+i v\right)(1+\cos 2 i v)}-\frac{1}{\pi} \int_{-\infty}^{\infty} \frac{\sin \left(2 t A_{N}-v \pi+2 t i v\right) d v}{\left(A_{N}+i v\right)(1+\cos 2 i v)} .
\end{aligned}
$$

Denote the right side of (3.12) by $J$ :

$$
|J|<\frac{\text { const. }}{A_{N}} \int_{0}^{\infty} \frac{1+\operatorname{ch} 2 t v}{\operatorname{ch} 2 v} d v=\frac{\text { const. }}{2 A_{N}} \pi+\frac{\text { const. }}{A_{N}} \frac{1}{\cos \frac{\pi t}{2}} .
$$

Then the limit of (3.11) becomes:

$$
\begin{aligned}
& \lim _{N \rightarrow \infty} \int_{0}^{1} R_{N}(t) f_{k}(t) d t=\lim _{N \rightarrow \infty} \int_{0}^{1} T_{N}(t) f_{k}(t) d t+\frac{1}{2 \pi i} \lim _{N \rightarrow \infty} \int_{0}^{1}\left(\int_{A_{N}-i \infty}^{A_{N}+i \infty} g(z) d z\right) f_{k}(t) d t \\
& =\lim _{N \rightarrow \infty} \int_{0}^{1} T_{N}(t) f_{k}(t) d t+\lim _{N \rightarrow \infty} \int_{0}^{A_{N}^{-1+\varepsilon}}\left(R_{N}(t)-T_{N}(t)\right) f_{k}(t) d t++\frac{1}{2 \pi i} \int_{A_{N}^{-1+\varepsilon}}^{1}\left[\int_{A_{N}-i \infty}^{A_{N}+i \infty} g(z) d z\right] f_{k}(t) .
\end{aligned}
$$


Using (3.6) and (3.13), we obtain

$$
\begin{aligned}
& \int_{A_{N}^{-1+\varepsilon}}^{1}\left|\left[\int_{A_{N}-i \infty}^{A_{N}+i \infty} g(z) d z\right] f_{k}(t)\right| d t \\
& \leq \lim _{N \rightarrow \infty} \frac{\text { const. }}{A_{N}} \int_{A_{N}^{-1+\varepsilon}}^{1}\left|f_{k}(t)\right| d t+\lim _{N \rightarrow \infty} \frac{\text { const. }}{A_{N}} \int_{A_{N}^{-1+\varepsilon}}^{1} \frac{\left|f_{k}(t)\right|}{\cos \frac{\pi t}{2}} d t=0 .
\end{aligned}
$$

Moreover, if (3.7) holds, then by virtue of the known relation for a large $N$ [[35], p. 642]

$$
T_{N}(t) \sim \frac{1}{2 t}\left[1-\frac{\sin 2 A_{N} t}{\sin \pi t}\right] .
$$

Hence, we will have

$$
\lim _{N \rightarrow \infty} \int_{0}^{A_{N}^{-1+\varepsilon}} T_{N}(t) f_{k}(t) d t=0
$$

Using property 2 and the asymptotic of $x_{m, k}$

$$
\lim _{N \rightarrow \infty} \int_{0}^{A_{N}^{-1+\varepsilon}} R_{N}(t) f_{k}(t) d t=0
$$

Earlier it was obtained that under the assumptions 1-3 (see [[7], Theorem 2.2])

$$
\lim _{N \rightarrow \infty} \int_{0}^{1} T_{N}(t) f_{k}(t) d t z=-\frac{2 v f_{k}(0)+f_{k}(1)}{4} .
$$

Thus, from (3.14) to (3.18), we have

$$
\lim _{N \rightarrow \infty} \int_{0}^{1} R_{N}(t) f_{k}(t) d t=-\frac{2 v f_{k}(0)+f_{k}(1)}{4} .
$$

Consequently,

$$
\sum_{k=N}^{\infty} \sum_{m=0}^{\infty} \int_{0}^{1} g\left(x_{m, k}\right) f_{k}(t) d t=\sum_{k=N}^{\infty}-\frac{2 v f_{k}(0)+f_{k}(1)}{4} .
$$

In a similar way to the one considered above, we get (this time Equation 2.5 has no imaginary roots, so the contour $C$ will only bypass the origin on the right half-plane):

$$
\sum_{k=1}^{N-1} \sum_{m=1}^{\infty} \int_{0}^{1} g\left(x_{m, k}\right) f_{k}(t) d t=-\sum_{k=1}^{N-1} \frac{2 v f_{k}(0)+f_{k}(1)}{4}
$$


Finally, combining (3.19) and (3.20), we get

$$
\sum_{i=1}^{\infty}\left(\mu^{(i)}-\lambda^{(i)}\right)=-\frac{2 v \operatorname{trq}(0)+\operatorname{trq}(1)}{4}
$$

which completes the proof.

Remark. It should be noted that in condition 1 , property $q^{(l)}(t) \in \sigma_{1}, l=\overline{0,2}$ may be weakened. Namely, we may just require to hold

$$
\sum_{j=1}^{\infty}\left|\left(q^{(l)}(t) \varphi_{j}, \varphi_{j}\right)\right|<\text { const. }
$$

Then formula (3.8) takes the form $\sum_{i=1}^{\infty}\left(\mu^{(i)}-\lambda^{(i)}\right)=-\frac{2 \nu \sum_{j=1}^{\infty}\left[\left(q(0) \varphi_{j}, \varphi_{j}\right)+\left(q(1) \varphi_{j}, \varphi_{j}\right)\right]}{4}$. There exist the bounded functions that are not from the trace class, even compact, but satisfy the above stated condition. Now, introduce an example.

Example. We consider the following boundary value problem:

$$
\begin{aligned}
& \frac{\partial u}{\partial t}=\frac{\partial^{2} u}{\partial x^{2}}+\frac{\partial^{2} u}{\partial y^{2}}-\frac{\partial^{4} u}{\partial z^{4}}-q(x, y, z) u, \quad t>0 \\
& \left(\left(1-\frac{h}{2}\right) u-h \frac{\partial u}{\partial n}-u_{t}\right)_{\partial \Omega \times[0,1]}=0 \\
& \left.u\right|_{z=0}=\left.u\right|_{z=1}=\left.\frac{\partial^{2} u}{\partial z^{2}}\right|_{z=0}=\left.\frac{\partial^{2} u}{\partial z^{2}}\right|_{z=1}=0
\end{aligned}
$$

in the cylinder $\partial \Omega \times[0,1]$, where $\Omega$ is a circle in $R^{2}\left((x, y) \in R^{2}\right)$ of radius 1 . Also, $\partial \Omega$ is a circumference of this circle, $n$ is an exterior normal to the surface $\partial \Omega \times[0,1]$ and $h=$ const.. Looking for the solution of this problem, which can be represented as $u(x, y, z, t)=U(x, y, z) T(t)$, we have

$$
\frac{T^{\prime}}{T}=\frac{\partial^{2} U}{\partial x^{2}} \cdot \frac{1}{U}+\frac{\partial^{2} U}{\partial y^{2}} \cdot \frac{1}{U}-\frac{1}{U} \frac{\partial^{4} U}{\partial z^{4}}-q(x, y, z) .
$$

Thus, the left-hand side of this equality depends only on $t$, while the right-hand side on $x, y, z$. This means they are equal to some constant which we will denote by $-\lambda$. Therefore,

$$
\frac{-\partial^{2} U}{\partial x^{2}}-\frac{\partial^{2} U}{\partial y^{2}}+\frac{\partial^{4} U}{\partial z^{4}}+q(x, y, z) U=\lambda U
$$

and (3.22) becomes like

$$
\left[\left(1-\frac{h}{2}\right) U-h \frac{\partial U}{\partial n}-\lambda U\right]_{\partial \Omega \times[0,1]}=0 .
$$

Using the cylindric coordinates $x=r \cos \phi, y=r \sin \phi, z=z$, we have

$$
\frac{-\partial^{2} U}{\partial r^{2}}-\frac{1}{r} \frac{\partial U}{\partial r}-\frac{1}{r^{2}} \frac{\partial^{2} U}{\partial \varphi^{2}}+\frac{\partial^{4} U}{\partial z^{4}}+q(r, \varphi, z) U=\lambda U .
$$


The boundary condition in (3.24) becomes

$$
\left(\left(1-\frac{h}{2}\right) U-h \frac{\partial U}{\partial r}-\lambda U\right)_{r=1}=0 .
$$

We will solve this problem by separation of variables. Taking $U(r, \phi, z)=V(r, z) \varphi$ $(\phi), q(r, \phi, z)=Q(r, z)$, and $\frac{\phi^{\prime \prime}}{\phi}=-v^{2}, v=$ const., we get

$$
\frac{-\partial^{2} V}{\partial r^{2}}-\frac{1}{r} \frac{\partial V}{\partial r}+\frac{\nu^{2}}{r^{2}} V+\frac{\partial^{4} V}{\partial z^{4}}+Q(r, z) V=\lambda V .
$$

By making $V(r, z)=\frac{V_{1}(r, z)}{\sqrt{r}}$ substitution, we get

$$
\frac{-\partial^{2} V_{1}}{\partial r^{2}}+\frac{v^{2}-\frac{1}{4}}{r^{2}} V_{1}+\frac{\partial^{4} V_{1}}{\partial z^{4}}+Q(r, z) V_{1}=\lambda V_{1}
$$

and (3.25), (3.23) take the form:

$$
\begin{gathered}
\left.\left(V_{1}(r, z)-h \frac{\partial V_{1}(r, z)}{\partial r}\right)\right|_{r=1}=\lambda V_{1}(1, z) \\
V_{1}(r, 0)=V_{1}(r, 1)=\left.\frac{\partial^{2} V_{1}}{\partial z^{2}}\right|_{z=0}=\left.\frac{\partial^{2} V_{1}}{\partial z^{2}}\right|_{z=1},
\end{gathered}
$$

where $Q(r, z)$ is a real-valued function which is continuous on $[0,1] \times[0,1]$, and has second partial derivative with respect to $r$ on $[0,1]$ for each fixed $z$. Fourier series of this function and its partial derivatives converge, respectively, to their values. Also assume that

$$
\int_{0}^{1} Q(0, z) d z=\int_{0}^{1} Q(1, z) d z=0 .
$$

Now, rewrite the problem in the differential operator form:

$$
\begin{gathered}
-v^{\prime \prime}(r)+\frac{v^{2}-\frac{1}{4}}{r^{2}} v(r)+A v(r)+q(r) v(r)=\lambda v(r) \\
v(1)-h v^{\prime}(1)=\lambda v(1)
\end{gathered}
$$

where $v(r)=V_{1}(r, \cdot)$ is a vector function with the values from $L_{2}(0,1)$. Operators $A$ and $q(r)$ are defined in the following way:

$$
\begin{gathered}
D(A)=\left\{u \in W_{2}(0,1) / u(0)=u(1)=u^{\prime \prime}(0)=u^{\prime \prime}(1)=0\right\}, \quad A u=\frac{\partial^{4} u}{\partial z^{4}}+\omega u, \quad \omega>0, \\
D(q(r))=L_{2}(0,1), \quad q(r) u=Q(r, z) u-\omega u .
\end{gathered}
$$

Obviously, the operator $A$ is self-adjoint, positive-definite, and $A^{-1}$ is a compact operator in $L_{2}(0,1)$. Also, the eigenvalues of $A$ are of the form:

$$
\mu_{k}(A)=\omega+\pi^{4} k^{4}, \quad k=1,2, \ldots
$$

Then, by virtue of Theorem 2.1, the eigenvalues of this problem behave like $\lambda_{m} \sim$ const. $m^{\frac{4}{3}}$. 
Using the statement of Theorem 3.2, we have

$$
\sum_{i=1}^{\infty}\left(\mu^{(i)}-\lambda^{(i)}\right)=-\frac{2 v \operatorname{trq}(0)+\operatorname{trq}(1)}{4}
$$

where $\mu_{i}$ are the eigenvalues of problem (3.28) with $q(r) \equiv 0$. Now calculate

$$
\begin{aligned}
\operatorname{trq}(0) & =2 \sum_{j=1}^{\infty} \int_{0}^{1} Q(0, z) \sin ^{2} j \pi z d z=\frac{2}{\pi} \sum_{j=1}^{\infty} \int_{0}^{\pi} Q\left(0, \frac{t}{\pi}\right) \sin ^{2} j t d t \\
& =\frac{2}{\pi} \sum_{j=1}^{\infty} \int_{0}^{\pi} Q\left(0, \frac{t}{\pi}\right) \frac{1-\cos 2 j t}{2} d t=-\sum_{j=1}^{\infty} \int_{0}^{\pi} Q\left(0, \frac{t}{\pi}\right) \cos 2 j t d t \\
& =\frac{1}{4}\left[\sum_{j=0}^{\infty} \cos j \cdot 0 \cdot \frac{2}{\pi} \int_{0}^{\pi} Q\left(0, \frac{t}{\pi}\right) \cos j t d t+\sum_{j=0}^{\infty} \cos j \pi \cdot \int_{0}^{\pi} Q\left(0, \frac{t}{\pi}\right) \cos j t d t\right] \\
& =\frac{1}{4}[Q(0,0)+Q(0,1)] .
\end{aligned}
$$

In a similar way, we can find

$$
\operatorname{trq}(1)=\frac{1}{4}[Q(1,0)+Q(1,1)]
$$

Therefore,

$$
\sum_{i=1}^{\infty}\left(\mu^{(i)}-\lambda^{(i)}\right)=-\frac{2 v[Q(0,0)+Q(0,1)]+Q(1,0)+Q(1,1)}{16} .
$$

The authors declare that they have no competing interests

\section{Acknowledgements}

The author would like to express his thanks to Dr. Yaghoob Ebrahimi, U.S.Fulbright Scholar assigned to Khazar University during 2009-10 academic year, for the latter's help in editing, interpretation, and modification of the initial version of this study.

\section{Author details}

'Department of Differential Equation, Institute of Mathematics and Mechanics-Azerbaijan National Academy of Science, 9, F. Agayev Street, Baku AZ1141, Azerbaijan ${ }^{2}$ Mathematics Department, Khazar University, Baku, Azerbaijan

Received: 2 December 2010 Accepted: 13 July 2011 Published: 13 July 2011

\section{References}

1. Gorbachuk, VI, Rybak, MA: On Boundary Value Problems for Sturm-Liouville Operator Equation with Eigenvalue Dependent Boundary Condition. Direct and Inverse Problems of Scattering Theory Kiev. 3-13 (1981)

2. Walter, J: Regular eigenvalue problems with eigenvalue parameter in the boundary conditions. Math Z. 133, 301-312 (1973). doi:10.1007/BF01177870

3. Fulton, ChT: Two-point boundary value problems with eigenvalue parameter contained in the boundary condition. Proc R Soc Edinburgh. 77A, 293-308 (1977)

4. Rybak, MA: On asymptotic of eigenvalue distribution of some boundary value problems for Sturm-Liouville operator equation. Ukr Math J. 32(2), 248-252 (1980)

5. Aliev, BA: Asymptotic behavior of eigenvalue of one boundary value problem for elliptic dif-operat. equation of second order. Ukr Math J. 58(8), 1146-1152 (2006)

6. Aslanova, NM: A trace formula of one boundary value problem for the Sturm-Liouville operator equation. Siberian Math J. 49(6), 1207-1215 (2008)

7. Hashimov, IF: Calculation of regularized trace for Sturm-Liouville operator with singularity on finite segment. M, DEP. VINITI, No 7340-B89. 37 (1989)

8. Maksudov, FG, Bayramogly, M, Adigezalov, AA: On regularized trace of Sturm-Liouville operator on finite segment with unbounded operator coefficient. DAN SSSR. 277(4), 795-799 (1984)

9. Sadovnichii, VA: On some identities for eigenvalues of singular differential operators. Relations for zeros of Bessel function. Westnik MGU, ser Math Mech. 3, 77-86 (1971) 
10. Meleshko, SV, Pokorniy, YV: On a vibrational boundary-value problem. Differ Equ. 23(8), 1466-1467 (1987)

11. Ahn, HJ: Vibrations of a pendulum consisting of a bob suspended from a wire. Quart Appl Math. 39(1), 109-117 (1981)

12. Racheva, MR: Bounds for the principal eigenvalue of nonhomogeneous bar with a tip mass. C.R Acad Bulgare Sci. 54(11), 23-26 (2001)

13. Kapustin, NY, Moiceev, El: On basicity in $L_{p}$ of a system of eigenfunctions responding to two problems with a spectral parameter in the boundary conditions. Differensialniye uravneniya. 36(10), 1357-1360 (2000)

14. Kapustin, NY, Moiceev, El: On pecularities of the root space of a spectral problem with a spectral parameter in the boundary condition. Docl RAN. 385(1), 20-24 (2002)

15. Kerimov, NB, Mirzoev, VS: On basis properties of one spectral problem with spectral parameter dependent boundary condition. Siberian Math J. 44(5), 1041-1045 (2003)

16. Yakubov, S: Solution of irregular problems by the asymptotic method. Asympt Anal. 22, 129-148 (2000)

17. Bayramoglu, M, Sahinturk, H: Higher order regularized trace formula for the regular Sturm-Liouville equation contained spectral parameter in the boundary condition. Appl Math Comput. 186(2), 1591-1599 (2007). doi:10.1016/j. amc.2006.08.066

18. Gelfand, IM, Levitan, BM: About one simple identity for eigenvalue of second order differential operator. DAN SSSR. 88(4), 593-596 (1953)

19. Aslanova, NM: Trace formula for Sturm-Liouville operator equation. Proc Math Mech Natl Acad Sci Azerb. XXVI, 53-61 (2007)

20. Sadovnichii, VA, Podolskii, VE: Trace of operators with relatively compact perturbation. Matem Sbor. 193(2), 129-152 (2002)

21. Dubrovskii, W: Abstract trace formulas for elliptic smooth differential operators given on compact manifolds. Diff Urav. 27(12), 2164-2166 (1991)

22. Halberg, CJA Jr, Kramer, VA: A generalization of the trace concept. Duke Math J. 27(4), 607-617 (1960). doi:10.1215/ S0012-7094-60-02758-7

23. Sadovnichii, VA, Podolskii, VE: Trace of operators. Uspech Math Nauk. 61(5), 89-156 (2006)

24. Dikii, LA: Trace formulas for Sturm-Liouville differential operators. Uspech Mathem Nauk XIII. 3(81), 111-143 (1958)

25. Sadovnichii, VA, Podolskii, VE: On the class of Sturm-Liouville operators and approximate calculation of first eigenvalues. Mat Sbornik. 189(1), 133-148 (1998)

26. Hochstadt, H: On the determination of a Hill's equation from its spectrum. Arch Rational Mech Anal. 19(5), 353-362 (1965)

27. Levitan, BM: Regularized trace and conditions for smooth periodicity for potential of Sturm-Liouville equation. Sib Matem J. 22(2), 137-148 (1981)

28. Fedosov, BV: About index of elliptic family on manifold with edge. Doklad AN SSSR. 248(5), 1066-1069 (1979)

29. Gesztesy, F, Simon, B: Topological invariance of the Witten index. J Funct Anal. 79(1), 91-102 (1988). doi:10.1016/00221236(88)90031-6

30. Gorbachuk, VI, Gorbachuk, ML: Boundary value problems for differential-operator equations. Nauk Dumka, Kiev, 284 (1984) (Russian).

31. Yakubov, S, Yakubov, Ya: Differential-Operator Equations Ordinary and Partial Differential Equations. Chapman and Hall/ CRC, Boca Raton, 568 (2000)

32. Lions, J-L, Magenes, E: Nonhomogeneous boundary value problems and their applications. Nauka, Moscow, 371 (1971)

33. Naymark, MA: Linear Differential Operators. Nauka, M. 528 (1969)

34. Smirnov, WI: Course of Mathematics. Nauka, M. 5, 655 (1959)

35. Watson, GN: A Treatise on the Theory of Bessel Function. IL, M. 1, 798 (1949)

36. Polya, G, Szego, G: Problems and Theorems from Analysis, II part. IL, M. 2, 431 (1978)

37. Gradstein, IS, Rijik, IM: Tables of Integrals, Sums, Series and Products. Nauka, M. 1108 (1971)

38. Gorbachuk, WI, Gorbachuk, ML: On some class of boundary value problems for Sturm-Liouville operator with operator potential. Ukr Mathem J. 24(3), 291-305 (1972)

doi:10.1186/1687-2770-2011-7

Cite this article as: Aslanova: Study of the asymptotic eigenvalue distribution and trace formula of a second order operator-differential equation. Boundary Value Problems 2011 2011:7.

\section{Submit your manuscript to a SpringerOpen ${ }^{\odot}$ journal and benefit from:}

- Convenient online submission

- Rigorous peer review

- Immediate publication on acceptance

- Open access: articles freely available online

- High visibility within the field

- Retaining the copyright to your article

Submit your next manuscript at $\gg$ springeropen.com 